\title{
Possible beneficial effect of bisphosphonates in osteonecrosis of the knee
}

\author{
Marius E. Kraenzlin • Christian Graf • \\ Christian Meier · Claude Kraenzlin • \\ Niklaus F. Friedrich
}

Received: 16 August 2009/Accepted: 2 March 2010/Published online: 8 April 2010

(C) Springer-Verlag 2010

\begin{abstract}
Osteonecrosis (ON) in the knee occurs as a localized inflammatory disease in relation to spontaneous or non-traumatic ON. Conservative treatment possibilities are limited, and prognosis appears to be poor; in most cases, ON results in knee arthroplasty. Bisphosphonates are suggested to prevent bone resorption and collapse of necrotic bone. In this observational, prospective study we investigated the effect of bisphosphonate treatment in patients with spontaneous or arthroscopy-induced ON of the knee. Twenty-eight patients with osteonecrotic lesions and bone marrow oedema in the knee were included. In 22 patients (80\%), ON was identified after arthroscopic surgery of the knee; six patients were diagnosed with spontaneous ON. Patients were initially given pamidronate $120 \mathrm{mg}$ i.v. divided in 3-4 perfusions over 2 weeks, followed by oral bisphosphonate treatment with alendronate $70 \mathrm{mg}$ weekly for 4-6 months. Bisphosphonate treatment resulted in a rapid pain relief, VAS decreasing from $8.2 \pm 1.2$ at baseline to $5.02 \pm 0.6$ after $4-6$ weeks $(p<0.001)$. After 6 months, the VAS decreased by $80 \%$ $(p<0.001)$. At the 6-month follow-up, symptoms had resolved completely in 15 patients out of 28; in 6 patients, minimal symptoms (VAS 1-2) remained. In two patients,
\end{abstract}

M. E. Kraenzlin · C. Meier

Division of Endocrinology, Diabetes and Clinical Nutrition,

University Hospital, 4031 Basel, Switzerland

C. Graf · N. F. Friedrich

Department of Orthopedic Surgery, Bruderholz Spital,

4101 Bruderholz, Switzerland

M. E. Kraenzlin $(\bowtie) \cdot$ C. Meier · C. Kraenzlin

Endocrine Practice and Laboratory, Missionsstrasse 24,

4055 Basel, Switzerland

e-mail: marius.kraenzlin@unibas.ch treatment effect was unsatisfactory, and surgical intervention was needed (arthroplasty). Bone marrow oedema on MRI resolved completely in 18 patients out of 28 with substantial reduction in the remaining. Furthermore, osteonecrotic area resolved completely or demarcation with sclerotic changes of the necrotic area could be observed. Bisphosphonate treatment in patients with osteonecrosis of the knee was associated with a rapid improvement in pain score and radiological consolidation of the area of osteonecrosis. Further randomized, controlled trials are warranted to confirm the potential beneficial role of bisphosphonates in the treatment of osteonecrosis of the knee. Level of evidence: observational study, level IV.

Keywords Osteonecrosis - Bisphosphonates · Arthroscopy · Knee

\section{Introduction}

Osteonecrosis (ON) is a localized inflammatory disease primarily affecting bone tissue. In its early stage, the overlaying articular cartilage is intact; however, with progression of the disease, structural failure in subchondral bone and overlaying cartilage tissue occurs [4, 7, 32, 40, 51]. Osteonecrosis of the knee is generally classified into two groups based on their underlying pathophysiological mechanisms [7, 15, 24, 40]. Spontaneous osteonecrosis of the knee (SONK) is characterized by a sudden onset of knee pain and affects predominately postmenopausal women [24, 40]. Although many causes have been proposed, the exact aetiology of ON of the knee is not entirely elucidated [17, 35, 39, 42, 46]. Primary or secondary vascular insufficiency leading to infarction of bone and minor, unrecognized traumatic incidents producing microfractures 
in the subchondral bone have been proposed $[17,35,39$, $42,46]$. Currently, the mechanical theory is favoured largely based on the finding of meniscal tears at the site of osteonecrotic condyle lesions. Histopathological studies support the theory of subchondral insufficiency fractures $[23,34,38,52]$. The second type of osteonecrosis, secondary or atraumatic osteonecrosis, is in most cases related to medical conditions such as corticosteroid treatment, sickle cell anaemia, systemic lupus erythematosus, hyperbarism and alcohol abuse [24, 40]. The onset of pain is rather gradual, and the disease occurs usually in patients under 50 years of age, predominantly women. In most cases, other joints are also involved, bilateral affection is very common. Similar to avascular necrosis of the hip, the underlying cause seems to be systemic and mostly of vascular origin.

Osteonecrosis of the knee after arthroscopy is a relatively new diagnostic entity and has been described in several reports $[6,10,12,13,20,23,26,35,39,42,46]$. In 49 cases, in which ON has been reported after arthroscopy, the development of osteonecrosis was diagnosed secondary to treatment of meniscal and chondral lesions or reconstruction of an intraarticular ligament. In some, bone lesions were thought to be in direct relation with meniscal or chondral lesions [10, 13, 15, 20, 26, 35, 39, 42, 46]. The prognosis of $\mathrm{ON}$ after arthroscopic surgery remains unclear but appears to be poorer than in ordinary SONK resulting in knee arthroplasty in many cases. Furthermore, conservative treatment options are limited and mostly already exhausted.

Bisphosphonates inhibit bone resorption and are widely used in metabolic bone disease characterized by increased osteoclast activity (i.e. osteoporosis, Paget's disease) [45]. In ON, structural failure is the result of resorption of necrotic bone during revascularisation, before new bone has formed. It can be hypothesized that if accelerated bone resorption could be reduced during the revascularization process until sufficient new bone has been formed, it would appear that structural failure with subsequent osteoarthritis could be avoided. In animal studies, it has been shown that bisphosphonate treatment prevents resorption of necrotic bone during revascularization and bone deformity $[8,9,27,30]$. In humans, bisphosphonate treatment has been used successfully in bone marrow oedema and avascular necrosis of the femoral head [3, 28, 29, 44, 49]. In these reports, the use of bisphosphonates was followed by a rapid improvement of clinical symptoms and radiological findings. To date, there is no report on the use of bisphosphonate treatment in $\mathrm{ON}$ of the knee. This observational, prospective study summarizes the effect of bisphosphonate treatment on the clinical and radiological course in patients with spontaneous or postarthroscopic ON of the knee.

\section{Materials and methods}

Study population

Twenty-eight patients with the radiologic finding of osteonecrotic lesions and bone marrow oedema in the knee were recruited into this open-label, prospective observational study. In 22 patients $(80 \%)$, ON was identified after arthroscopic surgery of the knee. Six patients were diagnosed with spontaneous osteonecrosis of the knee (SONK).

All patients included in the study had to be symptomatic and to meet the MRI diagnostic criteria. The criteria for diagnosis of ON on MRI included normal high-intensity signal of fat in the bone marrow replaced by a discrete lowintensity signal in the centre of the lesion and high-intensity signal area about the margin representing bone marrow oedema (Fig. 1). The size of the lesion was measured on T1-weighted images as the area of low signal intensity as described by Lotke et al. [32]. Informed consent to participate in the study was obtained from all patients, according to the rules of the local ethics committee.

\section{Intervention}

Patients were initially given pamidronate $120 \mathrm{mg}$ i.v. divided in 3-4 perfusions over 2 weeks, followed by oral bisphosphonate treatment with alendronate $70 \mathrm{mg}$ weekly for 4-6 months. This treatment regimen was chosen in analogy to experimental animal studies in which resorption of necrotic bone with alendronate treatment was prevented by doses $4-50$ times higher than the dose used in patients with osteoporosis. It was felt that an IV loading with bisphosphonate would therefore be more effective [8].

All patients were given non-steroidal anti-inflammatory or analgesics as needed. Weight-bearing was not forbidden but was usually avoided by the patients because of pain.

Calcium (500-1,000 mg/day) and vitamin D (400$800 \mathrm{IU} /$ day) supplements were provided throughout the whole treatment period.

\section{Measurements}

The initial evaluation included magnetic resonance imaging (MRI) in all patients and radiographs in a subset of patients, as well as a clinical and laboratory assessment.

Clinical assessments were made before treatment, 1 month later and thereafter at 6-12 weeks intervals. A further MRI scan was performed 3 months after initiating treatment.

Blood and urine samples were taken in the morning after an overnight fast. The baseline laboratory assessment 

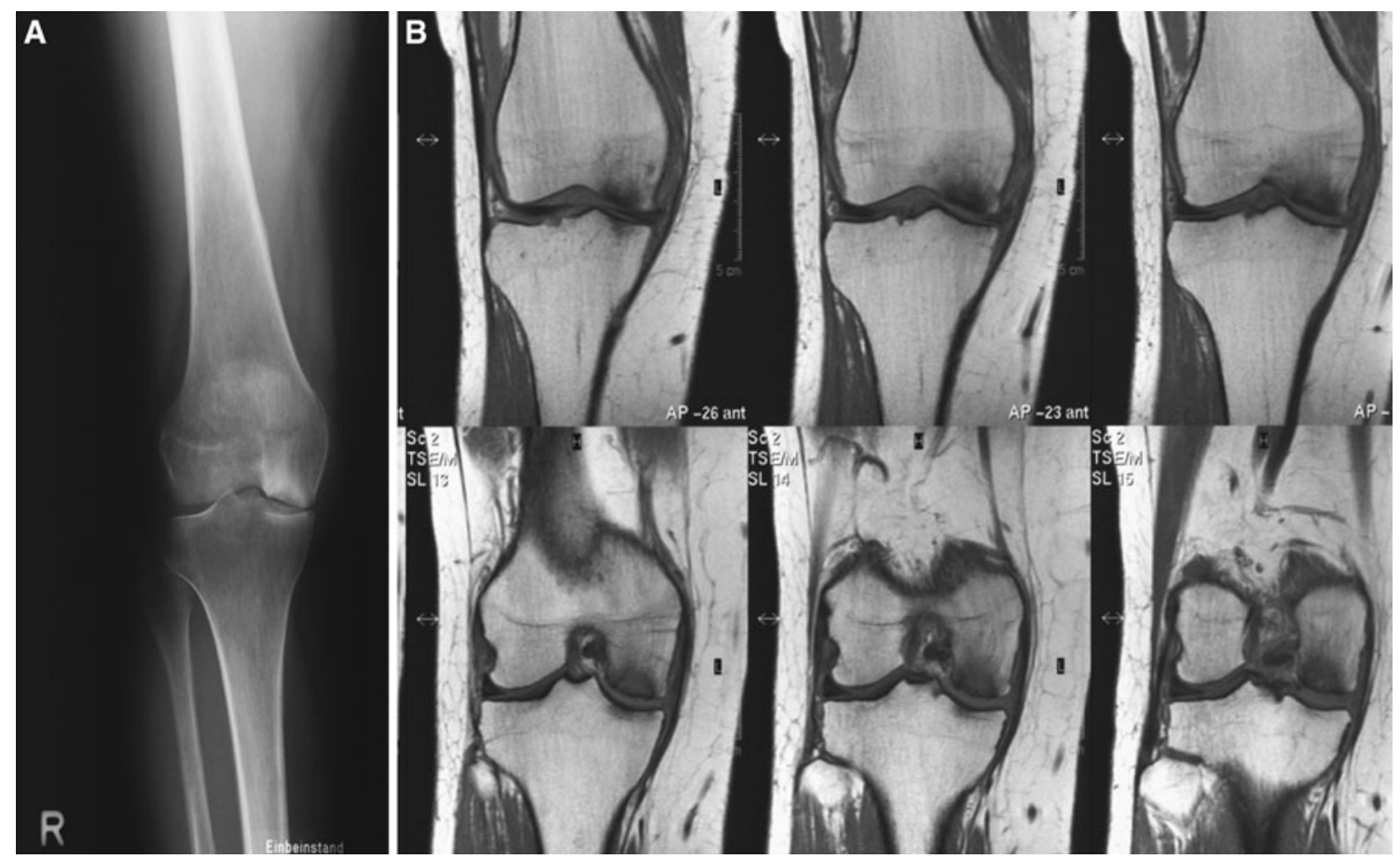

Fig. 1 X-ray (a) and magnetic resonance image (b) of a 62-year-old female patient from the series showing typical ostenecrotic lesion and oedema of the femoral condyle

included c-reactive protein, complete blood count, serum calcium, liver- and kidney function parameters, glucose, haemoglobin A1c and screening for coagulopathies.

Calcium, creatinine and total alkaline phosphatase (ALP) were analysed by standard method on an autoanalyser (Hitachi System 704 analyser; Roche Diagnostics, Rotkreuz, Switzerland). Serum bone alkaline phosphatise (BAP) concentration was determined by ELISA (ALKPHASE-B ELISA, Quidel) [22]. The parameters N-MIDOsteocalcin (OC) and intact parathyroid hormone (PTH) were measured in serum with electrochemiluminescence immunoassays (ECLIA) on the automated analyser Elecsys 2010 (Roche Diagnostics, Rotkreuz/Switzerland) [21, 47]. The intra- and interassay variation was $1.1-5.9 \%$ for OC, 2.6-6 and $1.7-5.5 \%$ for intact PTH. Urinary C-terminal teleopeptides of type I collagen was determined by ELISA (Nordic Bioscience Diagnostics A/S, Denmark) and corrected for creatinine [18]. The intra- and interassay CV's were 3.5 and $8.6 \%$, respectively. Pyridinium collagen cross-links (pyridinoline [PYD] and deoxypyridinoline [DPD]) were measured by high performance liquid chromatography (HPLC) using a commercially available kit from Chromsystems Instruments and Chemicals, Munich (Germany), and the results were expressed in relation to the urinary creatinine. The intra- and interassay were 8.3 and $11.5 \%$, respectively.

Pain was measured at baseline and during follow-up using a standardized visual analogue scale (VAS) [25].
Statistical analysis

All data are expressed as means \pm standard errors of the mean (SEM). To compare study variables during study period, one-way ANOVA for repeated measures with post hoc comparisons corrected for multiple testing by least squares difference (LSD) was used. Significance was defined as $p<0.05$. Data were analysed using Statistica for Windows (version 6.0; StatSoft, Tulsa, OK, USA).

\section{Results}

\section{Baseline characteristics}

The baseline characteristics are summarized in Table 1 . Twenty-two patients had an arthroscopy for meniscal lesions or osteoarthritis, and in six patients, ON developed spontaneously. The median time interval between arthroscopy or occurence of symptoms (in patients with SONK) and inclusion into the study was 4.0 months (range, 3 weeks to 24 months).

Laboratory investigations

Baseline laboratory investigations did not reveal any specific risk factor or disease associated with ON (Table 1). Bisphosphonate treatment resulted in an inhibition of bone 
Table 1 Baseline characteristics

\begin{tabular}{llc}
\hline & Normal range & \multicolumn{1}{l}{ Results } \\
\hline Age (years) & & $57.7 \pm 2.7$ \\
Height $(\mathrm{cm})$ & & $164.6 \pm 2.1$ \\
Weight $(\mathrm{kg})$ & & $75.7 \pm 3.3$ \\
Serum & & \\
Calcium $(\mathrm{mmol} / \mathrm{l})$ & $2.15-2.62$ & $2.43 \pm 0.02$ \\
Creatinine $(\mu \mathrm{mol} / \mathrm{l})$ & $<97$ & $73.4 \pm 3.2$ \\
Total ALP $(\mathrm{U} / \mathrm{L})$ & $35-104$ & $79.3 \pm 5.2$ \\
Bone ALP $(\mu \mathrm{g} / \mathrm{l})$ & $7.4-15.7$ & $13.8 \pm 2.1$ \\
Osteocalcin $(\mathrm{ng} / \mathrm{ml})$ & $7.7-32$ & $21.5 \pm 1.9$ \\
Intact PTH $(\mathrm{pg} / \mathrm{ml})$ & $10-60$ & $36.2 \pm 5.8$ \\
25-OH Vitamin D $(\mathrm{ng} / \mathrm{ml})$ & $>25$ & $18.7 \pm 3.6$ \\
Glucose $($ fasting) $(\mathrm{mmol} / \mathrm{l})$ & $<6.1$ & $5.3 \pm 0.6$ \\
HbA1c $(\%)$ & $<5.7$ & $5.1 \pm 0.7$ \\
Urine & & \\
Calcium/creatinine $(\mathrm{mmol} / \mathrm{mmol})$ & $0.1-0.32$ & $0.25 \pm 0.04$ \\
PYD/creatinine $(\mathrm{mmol} / \mathrm{mmol})$ & $60-100$ & $97.3 \pm 12.7^{*}$ \\
DPD/creatinine $(\mathrm{nmol} / \mathrm{mmol})$ & $9-20$ & $19.1 \pm 2.4^{*}$ \\
CTx/creatinine $(\mu \mathrm{g} / \mathrm{mmol})$ & $80-320$ & $281.6 \pm 38^{*}$ \\
\hline
\end{tabular}

Mean $\pm \mathrm{SEM} / * p<0.05$ compared to age-matched controls

turnover, as evidenced by a significant decrease in urinary levels of CTx, PYD and DPD (already after 4 weeks) and a decrease in serum concentrations of OC, TALP and BAP (after 3 months; Table 2).

Pain and disability

The mean VAS at baseline was $8.2 \pm 1.2$. As expected with such high VAS pain scores, quality of life and walking ability was severely reduced. Mobility was also affected, and patients could not walk a longer distance without walking aid.

Bisphosphonate treatment resulted in a rapid and substantial pain relief (Fig. 2). After 6 months of bisphosphonate therapy, the VAS decreased by $80 \%$ in average, and

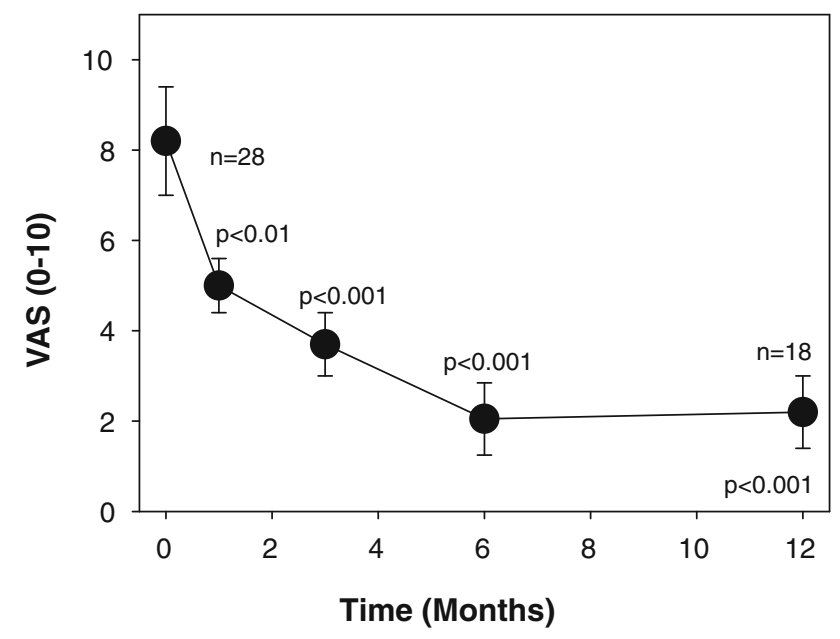

Fig. 2 Change in visual analogue scale pain scores $(V A S)$ in patients with osteonecrosis of the knee treated with bisphosphonates

all patients achieved a reduction of at least 50-60\%. At the 6-months follow-up in 15 patients out of 27 symptoms had resolved completely and in 6 patients complained of minimal residual pain (VAS 1-2). At 1 year follow-up, clinical data on 18 patients were available, and VAS was still reduced significantly $(2.2 \pm 0.8, p<0.001), 14$ patients reported to be free of symptoms. In 1 patient, there was evidence of moderate impression of the articular surface of the femoral condyle, however, as the patient was asymptomatic in his daily activities and in exercising (jogging) conservative treatment was continued. In two patients, treatment effect was unsatisfactory and surgical intervention (alloarthroplasty) was necessary.

Although no quantitative assessment of functional disability was performed, improvement in quality of life was apparent from the resolution of pain and considerable improvement in mobility was evident. All but two patients showed improvement in their walking ability without the needed of a walking aid.

Table 2 Biochemical parameters during follow-up

\begin{tabular}{lllll}
\hline & Baseline & 1 Month & 3 Months & 6 Months \\
\hline Calcium $(\mathrm{mmol} / \mathrm{l})$ & $2.44 \pm 0.02$ & $2.39 \pm 0.02$ & $2.35 \pm 0.04$ & $2.46 \pm 0.03$ \\
Creatinine $(\mu \mathrm{mol} / \mathrm{l})$ & $73.4 \pm 3.3$ & $75.2 \pm 3.4$ & $78.1 \pm 5.7$ & $81.9 \pm 6.3$ \\
ALP $(\mathrm{U} / \mathrm{L})$ & $79.3 \pm 5.3$ & $78.1 \pm 7.2$ & $80.8 \pm 11.0$ & $64.8 \pm 4.5^{*}$ \\
OC $(\mathrm{ng} / \mathrm{ml})$ & $21.5 \pm 2.0$ & $18.9 \pm 2.3$ & $12.5 \pm 1.7^{*}$ & $14.5 \pm 2.3^{*}$ \\
PYD $(\mathrm{nM} / \mathrm{mM}$ creatinine) & $97.3 \pm 12.7$ & $59.4 \pm 13.0^{*}$ & $44.9 \pm 4.2^{*}$ & $54.4 \pm 4.4^{*}$ \\
DPD $(\mathrm{nM} / \mathrm{mM}$ creatinine) & $19.0 \pm 2.4$ & $10.6 \pm 2.4^{*}$ & $8.2 \pm 0.7^{*}$ & $9.7 \pm 0.9^{*}$ \\
CTx $(\mu \mathrm{g} / \mathrm{mM}$ creatinine) & $231.6 \pm 38.5$ & $40.4 \pm 14.0^{*}$ & $52.3 \pm 14.7^{*}$ & $75.3 \pm 15.0^{*}$ \\
\hline
\end{tabular}

$A L P$ total alkaline phosphatase; $O C$ osteocalcin; $P Y D$ pyridinoline; $D P D$ deoxypyridinoline; $C T x$ c-terminal telopeptide

$* p<0.05$ compared to baseline 
Radiological findings

In all patients, the initial MRI scan showed typical appearance for $\mathrm{ON}$ with subchondral area of low signal and signal alteration indicative of perifocal bone oedema (low signal intensity on T1-weighted images corresponding to areas of high signal intensity on fat-suppressed T2weighted images). At the follow-up MRI after 2-3 months of treatment bone marrow oedema resolved completely in 18 patients (out of 27), in the remaining substantial reduction in bone oedema could be observed. The osteonecrotic area resolved completely in five patients; in the others, there was demarcation with sclerotic changes of the necrotic area. No further collapse of articular surface occurred during the whole observation period.

Tolarability and side effects

In general, bisphosphonate treatment was well tolerated. Four patients experienced an acute phase reaction after IV pamidronate with arthralgias and myalgias. The symptoms, however, were moderate and the treatment course could be completed in all patients. Oral treatment with alendronate was tolerated without side effects in all patients.

\section{Discussion}

The most important finding of the present study was the rapid and sustained improvement in clinical symptoms and disability, as well as resolution of the bone marrow oedema indicating a possible beneficial effect of bisphosphonates in patients with osteonecrosis. Specifically, pain reduction (assessed by the VAS) of 30\% after 4-6 weeks and of $80 \%$ after 6 months during bisphosphonate therapy could be observed. Complete or partial resolution of bone marrow oedema was documented in all cases. The size of the osteonecrotic area did not change significantly during the short observation period. However, in early stages of $\mathrm{ON}$, complete resolution and in more advanced stages, demarcation of the osteonecrotic area was observed.

The development of osteonecrosis following arthroscopic knee-surgery is a rare event. In a series of publications, a total of 49 cases have been reported [6, 10, 12, $13,20,23,26,35,39,42,46]$. In 21 patients, in which treatment outcome has been reported surgical reintervention (9 alloarthroplasties, 1 tibial osteotomy, 1 core decompression) was needed in eleven patients. Only in five cases a favourable outcome on conservative treatment (mechanical unloading, anti-inflammatory drugs) could be observed. From these observations one might conclude that favourable treatment response on non-invasive treatment is limited.
Prognosis appears to be unfavourable in postarthroscopic ON, with progression to irreversible stages in most cases [39]. Reasons for the poor outcome could be delayed diagnosis and treatment because of existing degenerative changes before the arthroscopic intervention. Treatment options of ON depend in general on the size of the lesion, smaller lesion being managed by mechanical unloading, insole with lateral wedge and use of non-steroidal anti-inflammatory drugs [39]. Larger lesions ( $>50 \%$ of the femoral condyle or $>5 \mathrm{~cm}^{2}$ ) in general require osteotomy or arthroplasty[31]. In early stages, treatment with core decompression of the bone has been successfully used [11, 19, 33]. Some authors report medical therapy with Iloprost, a prostacyclin analogue, which has been used in patients with bone marrow oedema and osteonecrosis, and in children with aseptic osteonecrosis of the proximal femur [5, 16, 41]. In these patients, treatment with Iloprost resulted in resolution of pain and rapid regression of the bone marrow oedema and subchondral lesions on MRI. In regard of the unfavourable prognosis and the limited non-surgical treatment possibility, other treatment possibilities are warranted in avoiding the need for arthroplasty.

In experimental animal studies, it has been shown that alendronate and more recently zoledronate treatment prevents resorption of necrotic bone during revascularization [8, 9]. There are several reports of successful bisphosphonate treatment of the transient osteoporosis of the hip or osteonecrosis of the femoral head [1, 2, 14, 29, 36, 37, 43, 50]. In an uncontrolled clinical study, 16 patients with avascular necrosis of the hip were treated with alendronate and an improvement in pain, disability and function compared to the expected natural history of the disease was found [3]. In a recently published observation from the same authors of 395 hips with avascular necrosis which were treated with alendronate for 3 years with a mean follow-up of 4 years improvement in the clinical function, a reduction in the rate of collapse and a decrease in the requirement for total hip replacement, compared with the findings of other studies in which no treatment was given [2]. In one of the few randomized controlled trial with alendronate treatment in 40 patients with atraumatic osteonecrosis of the femoral head with an involvement of at least $30 \%$ of the head, only one patient in the alendronate group needed a total hip arthroplasty whereas 16 subjects in the control group underwent total hip arthroplasty over an observation period of 28 months [29]. Thus, Alendronate treatment was effective in preventing early collapse of the femoral head in these patients. In another study using intravenous ibandronate in the treatment of transient osteoporosis of the hip, it has been shown that bone marrow oedema resolved completely in the MRI, and a significant pain relief could be observed [44]. 
In accordance with the findings in patients with avascular necrosis of the hip, bisphosphonates appear to be also beneficial in patients with osteonecrosis of the knee. The optimal dosage and duration of the bisphosphonate treatment in $\mathrm{ON}$ of the knee is not known. The dosage regimen used in the present study was chosen based on animal data which have shown that the prevention of resorption of necrotic bone during revascularisation is dose-dependent $[8,48]$. Nevertheless, this study would support the use of bisphosphonates for the treatment of osteonecrosis and bone marrow oedema of the knee.

There are limitations in this study. First, the number of patients included in the present study is rather small, nevertheless significant reduction in pain score could be detected early after initiation of bisphosphonate therapy. Secondly, the lack of a control group does not allow comparing definitively beneficial effects of bisphosphonates against the natural course of disease. And thirdly, the follow-up period was only 6-12 months, which may have been too short to demonstrate treatment failure.

\section{Conclusion}

In conclusion, bisphosphonate treatment of osteonecrosis of the knee was associated with a rapid improvement of the clinical symptoms and disability. Also complete or partial resolution of bone marrow oedema and demarcation or resolution of the osteonecrotic area in MRI could be observed. A preventive effect against progressive destruction of the architecture of the knee following spontaneous and postarthroscopic osteonecrosis can be assumed. Further randomized, controlled trials are needed to confirm a beneficial effect of bisphosphonate therapy in osteonecrosis of the knee.

\section{References}

1. Agarwala S, Jain D, Joshi VR, Sule A (2005) Efficacy of alendronate, a bisphosphonate, in the treatment of AVN of the hip. A prospective open-label study. Rheumatology (Oxford) 44:352-359

2. Agarwala S, Shah S, Joshi VR (2009) The use of alendronate in the treatment of avascular necrosis of the femoral head: follow-up to eight years. J Bone Jt Surg Br 91:1013-1018

3. Agarwala S, Sule A, Pai BU, Joshi VR (2002) Alendronate in the treatment of avascular necrosis of the hip. Rheumatol 41:346-347

4. Ahlback S, Bauer GC, Bohne WH (1968) Spontaneous osteonecrosis of the knee. Arthritis Rheum 11:705-733

5. Aigner N, Petje G, Schneider W, Meizer R, Wlk M, Kotsaris S, Knahr K, Landsiedl F (2005) Bone marrow edema syndrome of the femoral head: treatment with the prostacyclin analogue iloprost vs. core decompression: an MRI-controlled study. Wien Klin Wochenschr 117:130-135
6. Al Kaar M, Garcia J, Fritschy D, Bonvin JC (1997) Aseptic osteonecrosis of the femoral condyle after meniscectomy by the arthroscopic approach. J Radiol 78:283-288

7. Assouline-Dayan Y, Chang C, Greenspan A, Shoenfeld Y, Gershwin ME (2002) Pathogenesis and natural history of osteonecrosis. Semin Arthritis Rheum 32:94-124

8. Astrand J, Aspenberg P (2002) Systemic alendronate prevents resorption of necrotic bone during revascularization. A bone chamber study in rats. BMC Musculoskelet Disord 3:19-23

9. Astrand J, Harding AK, Aspenberg P, Tagil M (2006) Systemic zoledronate treatment both prevents resorption of allograft bone and increases the retention of new formed bone during revascularization and remodelling. A bone chamber study in rats. BMC Musculoskelet Disord 7:63

10. Athanasian EA, Wickiewicz TL, Warren RF (1995) Osteonecrosis of the femoral condyle after arthroscopic reconstruction of a cruciate ligament. Report of two cases. J Bone Jt Surg Am 77:1418-1422

11. Berger CE, Kroner AH, Kristen KH, Grabmeier GF, Kluger R, Minai-Pour MB, Leitha T, Engel A (2006) Transient bone marrow edema syndrome of the knee: clinical and magnetic resonance imaging results at 5 years after core decompression. Arthroscopy 22:866-871

12. Bonutti PM, Seyler TM, Delanois RE, McMahon M, McCarthy JC, Mont MA (2006) Osteonecrosis of the knee after laser or radiofrequency-assisted arthroscopy: treatment with minimally invasive knee arthroplasty. J Bone Jt Surg Am 88(Suppl 3):69-75

13. Brahme SK, Fox JM, Ferkel RD, Friedman MJ, Flannigan BD, Resnick DL (1991) Osteonecrosis of the knee after arthroscopic surgery: diagnosis with MR imaging. Radiology 178:851-853

14. Cardozo JB, Andrade DM, Santiago MB (2008) The use of bisphosphonate in the treatment of avascular necrosis: a systematic review. Clin Rheumatol 27:685-688

15. DeFalco RA, Ricci AR, Balduini FC (2003) Osteonecrosis of the knee after arthroscopic meniscectomy and chondroplasty: a case report and literature review. Am J Sports Med 31:1013-1016

16. Disch AC, Matziolis G, Perka C (2005) The management of necrosis-associated and idiopathic bone-marrow oedema of the proximal femur by intravenous iloprost. J Bone Jt Surg $\mathrm{Br}$ 87:560-564

17. Faletti C, Robba T, de Petro P (2002) Postmeniscectomy osteonecrosis. Arthroscopy 18:91-94

18. Fledelius C, Kolding I, Bonde M, Cloos P, Christgau S (1996) Specificity of the crosslaps ELISA and the MabA7 ELISA. Osteoporosis Int 6(Suppl 1):PMO440

19. Forst J, Forst R, Heller K-D, Adam G (1998) Spontaneous osteonecrosis of the femoral condyle: causal treatment by early core decompression. Arch Orthop Trauma Surg 117:18-22

20. Garino JP, Lotke PA, Sapega AA, Reilly PJ, Esterhai JL Jr (1995) Osteonecrosis of the knee following laser-assisted arthroscopic surgery: a report of six cases. Arthroscopy 11:467-474

21. Garnero P, Borel O, Delmas P (2001) Evaluation of a fully automated serum assay for c-terminal cross-linking teleopeptide of type I collagen in osteoporosis. Clin Chem 47:694-702

22. Gomez B Jr, Ardakani S, Ju J, Jenkins D, Cerelli MJ, Daniloff YN, Kung VT (1995) Monoclonal antibody assay for measuring bone-specific alkaline phosphatase activity in serum. Clin Chem 41:1560-1566

23. Hall FM (2005) Osteonecrosis in the postoperative knee. Radiology 236:370-371

24. Hofmann S, Kramer J, Vakil-Adli A, Aigner N, Breitenseher M (2004) Painful bone marrow edema of the knee: differential diagnosis and therapeutic concepts. Orthop Clin North Am 35:321-333

25. Huskisson EC (1974) Measurement of pain. Lancet 2:1127-1131 
26. Johnson TC, Evans JA, Gilley JA, DeLee JC (2000) Osteonecrosis of the knee after arthroscopic surgery for meniscal tears and chondral lesions. Arthroscopy 16:254-261

27. Kim HKW, Randall TS, Bian H, Jenkins J, Garces A, Bauss F (2005) Ibandronate for prevention of femoral head deformity after ischemic necrosis of the capital femoral epiphysis in immature pigs. J Bone Jt Surg 87:550-557

28. La Montagna G, Malesci D, Tirri R, Valentini G (2005) Successful neridronate therapy in transient osteoporosis of the hip. Clin Rheumatol 24:67-69

29. Lai KA, Shen WJ, Yang CY, Shao CJ, Hsu JT, Lin RM (2005) The use of alendronate to prevent early collapse of the femoral head in patients with nontraumatic osteonecrosis. A randomized clinical study. J Bone Jt Surg Am 87:2155-2159

30. Little DG, Peat RA, McEvoy A, Williams PR, Smith EJ, Baldock PA (2003) Zoledronic acid treatment results in retention of femoral head structure after traumatic osteonecrosis in young wistar rats. J Bone Miner Res 11:2016-2022

31. Lotke PA, Battish R, Nelson CL (2001) Treatment of osteonecrosis of the knee. Instr Course Lect 50:483-488

32. Lotke PA, Ecker ML (1988) Osteonecrosis of the knee. J Bone Jt Surg Am 70:470-473

33. Marulanda G, Seyler TM, Sheikh NH, Mont MA (2006) Percutaneous drilling for the treatment of secondary osteonecrosis of the knee. J Bone Jt Surg Br 88:740-746

34. Muscolo DL, Costa-Paz M, Ayerza M, Makino A (2006) Medial meniscal tears and spontaneous osteonecrosis of the knee. Arthroscopy 22:457-460

35. Muscolo DL, Costa-Paz M, Makino A, Ayerza MA (1996) Osteonecrosis of the knee following arthroscopic meniscectomy in patients over 50-years old. Arthroscopy 12:273-279

36. Nguyen T, Zacharin MR (2006) Pamidronate treatment of steroid associated osteonecrosis in young patients treated for acute lymphoblastic leukaemia-two-year outcomes. J Pediatr Endocrinol Metab 19:161-167

37. Nishii T, Sugano N, Miki H, Hashimoto J, Yoshikawa H (2006) Does alendronate prevent collapse in osteonecrosis of the femoral head? Clin Orthop Relat Res 443:273-279

38. Norman A, Baker ND (1978) Spontaneous osteonecrosis of the knee and medial meniscal tears. Radiology 129:653-656

39. Pape D, Seil R, Anagnostakos K, Kohn D (2007) Postarthroscopic osteonecrosis of the knee. Arthroscopy 23:428-438
40. Patel DV, Breazeale NM, Behr CHT, Warren RF, Wickiewicz TL, O'Brien SJ (1998) Osteonecrosis of the knee: current clinical concepts. Knee Surg Sports Traumatol Arthrosc 6:2-11

41. Petje G, Radler C, Aigner N, Manner H, Kriegs-Au G, Grill F (2004) Pharmacological management of aseptic osteonecrosis in children. Expert Opin Pharmacother 5:1455-1462

42. Prues-Latour V, Bonvin JC, Fritschy D (1998) Nine cases of osteonecrosis in elderly patients following arthroscopic meniscectomy. Knee Surg Sports Traumatol Arthrosc 6:142-147

43. Ramachandran M, Ward K, Brown RR, Munns CF, Cowell CT, Little DG (2007) Intravenous bisphosphonate therapy for traumatic osteonecrosis of the femoral head in adolescents. J.Bone Jt Surg Am 89:1727-1734

44. Ringe JD, Dorst A, Faber H (2005) Effective and rapid treatment of painful localized transient osteoporosis (bone marrow edema) with intravenous ibandronate. Osteoporos Int 16:2063-2068

45. Russell RG (2006) Bisphosphonates: from bench to bedside. Ann NY Acad Sci 1068:367-401

46. Santori N, Condello V, Adriani E, Mariani PP (1995) Osteonecrosis after arthroscopic medial meniscectomy. Arthroscopy $11: 220-224$

47. Schmidt-gayk H, Spanuth E, Kotting J, Bartl R, Felsenberg D, Pfeilschifter J, Raue F, Roth HJ (2004) Performance evaluation of automated assays for beta-crosslaps, N-MID-osteocalcin and intact parathyroid hormone (BIOROSE multicenter study). Clin Chem Lab Med 42:90-95

48. Tagil M, Astrand J, Westman L, Aspenberg P (2004) Alendronate prevents collapse in mechanically loaded osteochondral grafts: a bone chamber study in rats. Acta Orthop Scand 75:756-761

49. Varenna M, Zucchi F, Binelli L, Failoni S, Gallazzi M, Sinigaglia L (2002) Intravenous pamidronate in the treatment of transient osteoporosis of the hip. Bone 31:96-101

50. Wang CJ, Wang FS, Yang KD, Huang CC, Lee MS, Chan YS, Wang JW, Ko JY (2008) Treatment of osteonecrosis of the hip: comparison of extracorporeal shockwave with shockwave and alendronate. Arch Orthop Trauma Surg 128:901-908

51. Watson RM, Roach NA, Dalinka MK (2004) Avascular necrosis and bone marrow edema syndrome. Radiol Clin North Am 42:207-219

52. Yamamoto T, Bullough PG (2000) Spontaneous osteonecrosis of the knee: the result of subchondral insufficiency fracture. J Bone Jt Surg Am 82:858-866 